\title{
Acid fibroblast growth factor facilitates the progression of atherosclerotic plaques regardless of alterations in serum lipid expression levels in HFD-fed $\mathrm{ApoE}^{-/-}$mice
}

\author{
JIBO HAN ${ }^{1,2^{*}}$, YAO DU $^{3^{*}}$, LINTAO WANG $^{2},{\text { XIONG } \text { CHEN }^{2}, \text { LIQIN JIANG }^{1} \text { and JIANJIANG XU }}^{1}$ \\ ${ }^{1}$ Department of Cardiology, The Second Affiliated Hospital of Jiaxing University, Jiaxing, Zhejiang 314000; \\ ${ }^{2}$ Chemical Biology Research Center, School of Pharmaceutical Sciences, Wenzhou Medical University, Wenzhou, \\ Zhejiang 325000; ${ }^{3}$ Medication Department, Nanjing Drum Tower Hospital Affiliated to \\ Medical College of Nanjing University, Nanjing, Jiangsu 210000, P.R. China
}

Received December 11, 2017; Accepted April 24, 2018

DOI: $10.3892 / \mathrm{mmr} .2018 .9060$

\begin{abstract}
Atherosclerosis is recognized at present as a chronic metabolic disease of the arteries that leads to multifocal plaque development. Previous studies have reported that acid fibroblast growth factor (aFGF) is a critical therapeutic regulator in numerous chronic metabolic disorders. However, there is currently no direct evidence indicating whether aFGF serves a therapeutic role in atherosclerosis. In the present study, the role of aFGF in atherosclerotic lesion development was investigated by examining high-fat diet (HFD)-fed apolipoprotein E $(\text { ApoE })^{-/-}$mice and parenteral administration of aFGF. Increased expression of aFGF and peroxisome proliferator-activated receptor $\alpha(\operatorname{PPAR} \alpha)$ was observed during atherosclerotic lesion development. The parenteral delivery of aFGF facilitated the progression of atherosclerosis without altering serum lipid expression levels in HFD-fed $\mathrm{ApoE}^{-/-}$mice. Furthermore, it was demonstrated that aFGF increased the expression of PPAR $\alpha$ and inflammatory cytokines. The present results provided evidence that aFGF accelerates the progression of atherosclerosis and suggested that aFGF may be a potential therapeutic target for the prevention of atherosclerosis development.
\end{abstract}

\section{Introduction}

Acid fibroblast growth factor 1 (aFGF) is a mitogenic factor that has been associated with peroxisome proliferator-activated

Correspondence to: Dr Liqin Jiang or Dr Jianjiang Xu, Department of Cardiology, The Second Affiliated Hospital of Jiaxing University, 1518 Huancheng North Road, Jiaxing, Zhejiang 314000, P.R. China E-mail: lqjiangjx2y@163.com

E-mail: jjxvjx2y@163.com

*Contributed equally

Key words: atherosclerosis, acid fibroblast growth factor, peroxisome proliferator-activated receptor $\alpha$, inflammatory response receptors (PPARs) $(1,2)$, has been reported to be a critical therapeutic regulator in numerous chronic metabolic disorders. aFGF-knockout mice develop insulin resistance when stressed with a high-fat diet (HFD), suggesting that aFGF has a beneficial effect on nutrient homeostasis (1). aFGF additionally has therapeutic potential for the treatment of non-alcoholic fatty liver disease (2).

Atherosclerosis is the principal cause of coronary artery disease, and is therefore a principal cause of mortality and morbidity globally (3). It is noteworthy that atherosclerosis is additionally recognized as a lipid-driven chronic metabolic disease (4). Previous studies have reported increased aFGF expression in atherosclerotic plaques in human (5) and swine (6) models. However, there is no direct evidence indicating whether aFGF serves a therapeutic role in atherosclerosis. Recent studies demonstrated that PPAR $\alpha$ is a key regulator of atherosclerosis and is involved in HFD-induced atherosclerosis in apolipoprotein E (ApoE)-null mice (7).

In the present study, the role of increased aFGF and PPAR $\alpha$ expression in atherosclerotic lesion development was verified by examining ApoE-null mice. Furthermore, it was identified that parenteral delivery of aFGF increased the expression of PPAR $\alpha$, the induction of inflammatory cytokines, and the subsequent development of atherosclerotic plaques.

\section{Materials and methods}

Animal experiments. The protocols used for all animal studies were approved by the Wenzhou Medical University Animal Policy and Welfare Committee (Wenzhou, China; approval no. wydw2014-0058). Male ApoE $^{-/-}$mice $(n=28 ; 18-20$ g; 8 weeks old) with a C57BL/6 background were purchased from Beijing HFK Bioscience Co., Ltd., (Beijing, China). Mice were housed at $22 \pm 2.0^{\circ} \mathrm{C}$ with $50 \pm 5 \%$ humidity in a $12 \mathrm{~h}$ light/dark cycle with free access to food and water. To induce atherosclerosis, the mice were fed a HFD containing $60 \% \mathrm{kcal}$ from fat, $20 \% \mathrm{kcal}$ from protein and $20 \% \mathrm{kcal}$ from carbohydrate (MediScience Diets Co., Ltd., Yangzhou, China; cat. no. MD12033) for 16 weeks (n=7; ApoE HFD), while the control animals were fed a normal-fat diet (NFD) containing 
$10 \% \mathrm{kcal}$ from fat, $20 \% \mathrm{kcal}$ from protein and $70 \% \mathrm{kcal}$ from carbohydrate (MediScience Diets Co., Ltd.; cat. no. MD12031; $\mathrm{n}=7$; ApoE NFD).

In the second set of experiments, mice that were fed a HFD for 8 weeks were randomly divided into the following two groups: ApoE HFD treated with vehicle via intraperitoneal (IP) injection (PBS for 8 weeks; $n=7$ ) and ApoE HFD treated with aFGF (Key Laboratory of Biotechnology and Pharmaceutical Engineering, Zhejiang, China) via IP injection $[0.5 \mathrm{mg} / \mathrm{kg} / 2$ days for 8 weeks, as described in a previous paper (2); $n=7]$. The mice were sacrificed by increasing $\mathrm{CO}_{2}$ inhalation, in accordance with Schedule 1 of the Animals (Scientific Procedures) Act (1986) as previously described (8), and blood was collected into a syringe containing $4 \%$ trisodium citrate $(1: 10, \mathrm{v} / \mathrm{v})$ via cardiac puncture. Arterial tissues were fixed in $4 \%$ paraformaldehyde at a room temperature for $24 \mathrm{~h}$ and embedded in optimum cutting temperature compound. Tissues were snap-frozen in liquid nitrogen and serial $10 \mu \mathrm{m}$-thick cryosections from the middle portion of the tissues were collected for gene and protein expression analysis.

Measurement of the expression levels of serum lipids and biochemical indicators. The expression level of serum lipids was measured using lipid-specific biochemical kits [Nanjing Jiancheng Bioengineering Institute, Nanjing, China; cat. no. A110-1 for triglycerides (TG); cat. no. A113-1 for low-density lipoprotein (LDL); cat. no. A112-1 for high density lipoprotein (HDL); and cat. no. F002-1 for total cholesterol (TC)].

Immunofluorescence staining. The expression of aFGF and PPAR $\alpha$ was measured by immunofluorescence staining. Frozen sections were used for immunofluorescence analysis. The slides were blocked using $1 \%$ bovine serum albumin for $30 \mathrm{~min}$ at room temperature and incubated overnight at $4^{\circ} \mathrm{C}$ with an aFGF antibody (1:200; cat. no. ab169748) or a PPAR $\alpha$ antibody (1:200; cat. no. ab119416). A tetramethylrhodamine-conjugated secondary antibody (1:200; cat. no. ab6786; all Abcam, Cambridge, UK) was used for detection at $4^{\circ} \mathrm{C}$ for $1 \mathrm{~h}$. The slides were additionally stained with DAPI at $4^{\circ} \mathrm{C}$ for $5 \mathrm{~min}(5 \mathrm{mg} / \mathrm{ml}$; Beyotime Institute of Biotechnology, Haimen, China; cat. no. C1005). Slides were viewed under a fluorescence microscope (magnification, $\mathrm{x} 200$ ). The images were analyzed with Image-Pro Plus (version 6.0 Media Cybernetics, Inc., Rockville, MD, USA).

Histology and analysis of atherosclerotic lesions. Atherosclerotic lesions were measured as described in a previous paper (9). The whole aorta, including the aortic arch and the thoracic and abdominal segments, was dissected, gently cleaned of adventitial tissue and stained with Oil Red O at room temperature for $15 \mathrm{~min}(5 \mathrm{mg} / \mathrm{ml}$; Nanjing Jiancheng Bioengineering Institute; cat. no. D027). The surface lesion area was quantified with ImageJ software (version 1.6.2; National Institutes of Health, Bethesda, MD, USA). To measure lesions in the aortic root, the heart and proximal aorta were excised, and the apex and lower half of the ventricles were removed and stained with Oil Red O for $15 \mathrm{~min}(5 \mathrm{mg} / \mathrm{ml})$ at room temperature. The surface lesion area was quantified with ImageJ software.
Five frozen sections were also stained with hematoxylin and eosin at room temperature (eosin for $2 \mathrm{~min}$ and hematoxylin for $5 \mathrm{~min}$ ) for histopathological observation.

Reverse transcription-quantitative polymerase chain reaction (RT-qPCR). Total RNA was isolated from arterial tissues using TRIzol ${ }^{\circledR}$ (cat. no. 15596026). RT and qPCR were performed using a two-step Moloney Murine Leukemia Virus kit (cat. no. 28025013) and a Platinum SYBR Green qPCR SuperMix-uracil DNA glycosylase kit (cat. no. 11733046; Thermo Fisher Scientific, Inc., Waltham, MA, USA) in an Eppendorf Mastercycler ep RealPlex detection system (Eppendorf, Hamburg, Germany). PCR quantification was performed using the $2^{-\Delta \Delta \mathrm{Ca}}$ method (10). Primers were obtained from Thermo Fisher Scientific, Inc. The primer sequences are listed in Table I. mRNA expression levels of the target genes were normalized to $\beta$-actin.

Statistical analysis. Data are presented as the mean \pm standard error of the mean. Differences between the groups were determined using the Student's t-test, as appropriate, in GraphPad Prism 5.01 (GraphPad Software Inc., La Jolla, CA, USA). $\mathrm{P}<0.05$ was considered to indicate a statistically significant difference.

\section{Results}

Increased expression of $a F G F$ and PPAR in the aortas of HFD-fed ApoE $E^{-1}$ mice. A classical paradigm of the HFD-induced $\mathrm{ApoE}^{-/-}$atherosclerosis model is the alteration of serum lipid expression levels, as elevated LDL has been demonstrated to be strongly associated with the development of atherosclerosis (8). ApoE ${ }^{-1-}$ mice fed a HFD exhibited significantly increased serum expression levels of TG, TC and LDL (Fig. 1A-C; $\mathrm{P}<0.001$ ) and significantly reduced expression levels of high-density lipoprotein (HDL) compared with control mice fed an NFD (Fig. 1D; P<0.05).

The aortic tissues from the mice were subsequently assessed by immunofluorescence staining to determine whether aFGF and PPAR $\alpha$ are involved in the progression of atherosclerotic plaques. The expression levels of aFGF (Fig. 2A) and PPAR $\alpha$ (Fig. 2B) were increased in HFD-fed mice compared with NFD-fed mice in atherosclerotic lesions of the aortic root and aorta. The mRNA isolated from aortic tissues confirmed that the expression levels of aFGF were increased in HFD-fed mice (Fig. 2C). These increased expression levels of aFGF and PPAR $\alpha$ corresponded with morphological alterations in HFD-fed $\mathrm{ApoE}^{-/}$mice, including an augmented atherosclerotic plaque lesion area in the aortic root (Fig. 2D) and aorta (Fig. 2E) compared with ApoE NFD mice, reinforcing the hypothesis that there is a positive association between atherosclerotic plaque development and increased aFGF and PPAR $\alpha$ expression levels.

Treatment with aFGF aggravates atherosclerotic plaque development in HFD-fed ApoE-/ mice. The second set of experiments aimed to determine whether parenteral administration of aFGF was associated with HFD-induced atherosclerotic development. Oil Red O staining of the entire aorta was performed in the en face preparation and of the aortic 


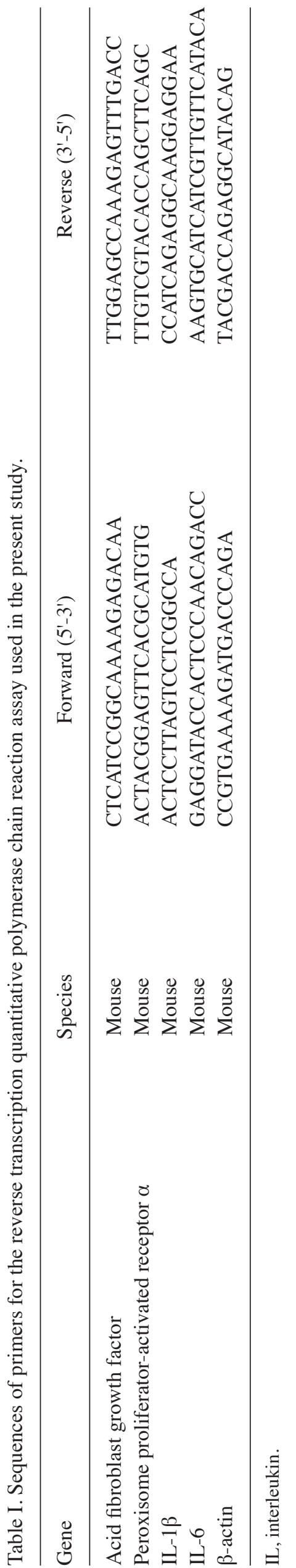

root to measure the severity of these lesions. Notably, the present results demonstrated a significantly increased lesion area in ApoE HFD mice treated with aFGF compared with ApoE HFD mice treated with vehicle in the entire aorta (Fig. 3A and B) and the aortic root (Fig. 3C and D). An additional assessment by hematoxylin and eosin staining demonstrated that the plaque areas in the aortic root of aFGF-treated mice were aggravated in comparison with vehicle-treated HFD-fed mice (Fig. 3E). The present results indicated that the administration of aFGF accelerated the progression of atherosclerotic plaques.

Treatment of mice with aFGF does not affect the expression levels of serum lipids. The present study additionally aimed to determine whether the administration of aFGF alters serum lipid expression levels. Notably, the treatment of mice with aFGF for 8 weeks did not affect the expression levels of serum lipids, including TG (Fig. 4A), TC (Fig. 4B), LDL (Fig. 4C) and HDL (Fig. 4D), compared with vehicle-treated HFD-fed mice, suggesting that the aFGF-facilitated progression of atherosclerosis may be trigged or maintained via mechanisms that are parallel to or independent of hyperlipidaemia.

Administration of aFGF increases the mRNA expression levels of PPAR $\alpha$ and inflammatory factors in HFD-fed ApoE ${ }^{-/-}$mice. It has been established that PPAR $\alpha$ (7) and inflammation (11) contribute to atherosclerotic lesions. To determine whether the aggravating effect of aFGF on atherogenesis is associated with PPAR $\alpha$ and its downstream inflammatory factors (12), the mRNA expression levels of PPAR $\alpha$ and associated inflammatory factors were assessed, including interleukin (IL)-1 $\beta$ and IL-6, in vivo. The present data demonstrated that the mRNA expression levels of PPAR $\alpha$ (Fig. 4E), IL-6 (Fig. 4F) and IL-1 $\beta$ (Fig. 4G) all significantly increased when atherosclerotic mice were treated with aFGF $(\mathrm{P}<0.05)$.

\section{Discussion}

Atherosclerosis is a systemic, chronic metabolic disease of the principal arteries. The formation and progression of atherosclerotic plaques involves hyperlipidemia, inflammation, foam cell formation, smooth muscle cell proliferation and increased matrix synthesis (13). In the present study, it was observed that atherosclerosis was associated with elevated aFGF expression levels, consistent with a previous report (14).

The FGF family, which contains 22 members in mammals, has diverse biological functions in the progression of atherosclerotic plaques (15). Basic (b)FGF has been detected in human atherosclerotic plaques (16), and increased expression of bFGF is associated with carotid atherosclerotic plaque instability (17). By contrast, the depletion of FGF21 in $\mathrm{ApoE}^{-/-}$mice results in a markedly increased exacerbation of atherosclerosis, which may be reversed by replenishment with exogenous mouse recombinant FGF21 (18). The key present results suggested that aFGF facilitated the progression of atherosclerosis regardless of alterations in serum lipid expression levels, which is inconsistent with the protective role of aFGF in other chronic metabolic diseases $(1,2)$. However, FGFs exert their biological effects by interacting with and activating FGF receptors (FGFRs) (14). The present results are consistent with those reported by Raj et al (14), who demonstrated 

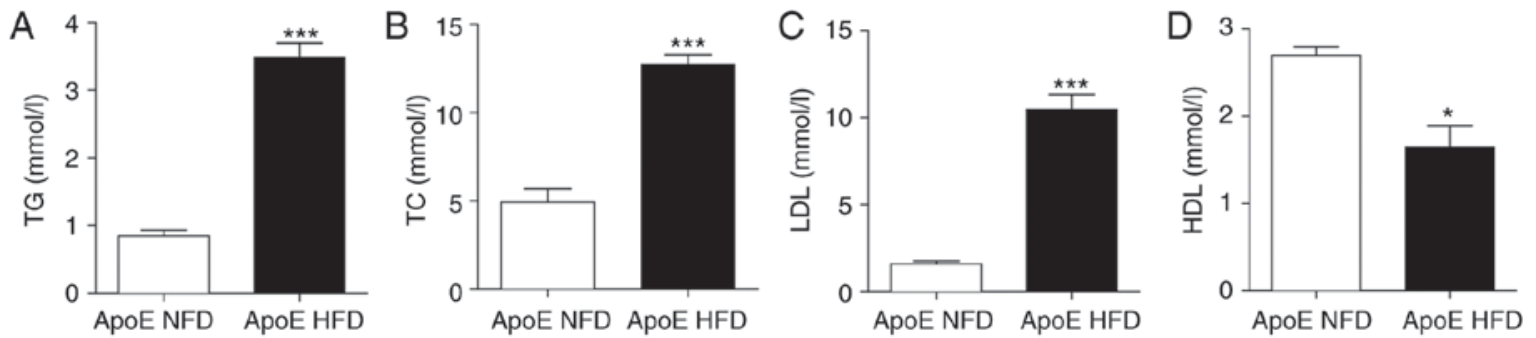

Figure 1. ApoE ${ }^{-/}$mice were placed on a HFD and examined following 16 weeks. Serum levels of (A) TG, (B) TC, (C) LDL and (D) HDL were analyzed. $\mathrm{n}=7 /$ group; ${ }^{*} \mathrm{P}<0.05,{ }^{* * * *} \mathrm{P}<0.001$ vs. respective ApoE NFD. ApoE, apolipoprotein E; HFD, high-fat diet; TG, triglycerides; TC, total cholesterol; LDL, low-density lipoprotein; HDL, high-density lipoprotein; NFD, normal-fat diet.
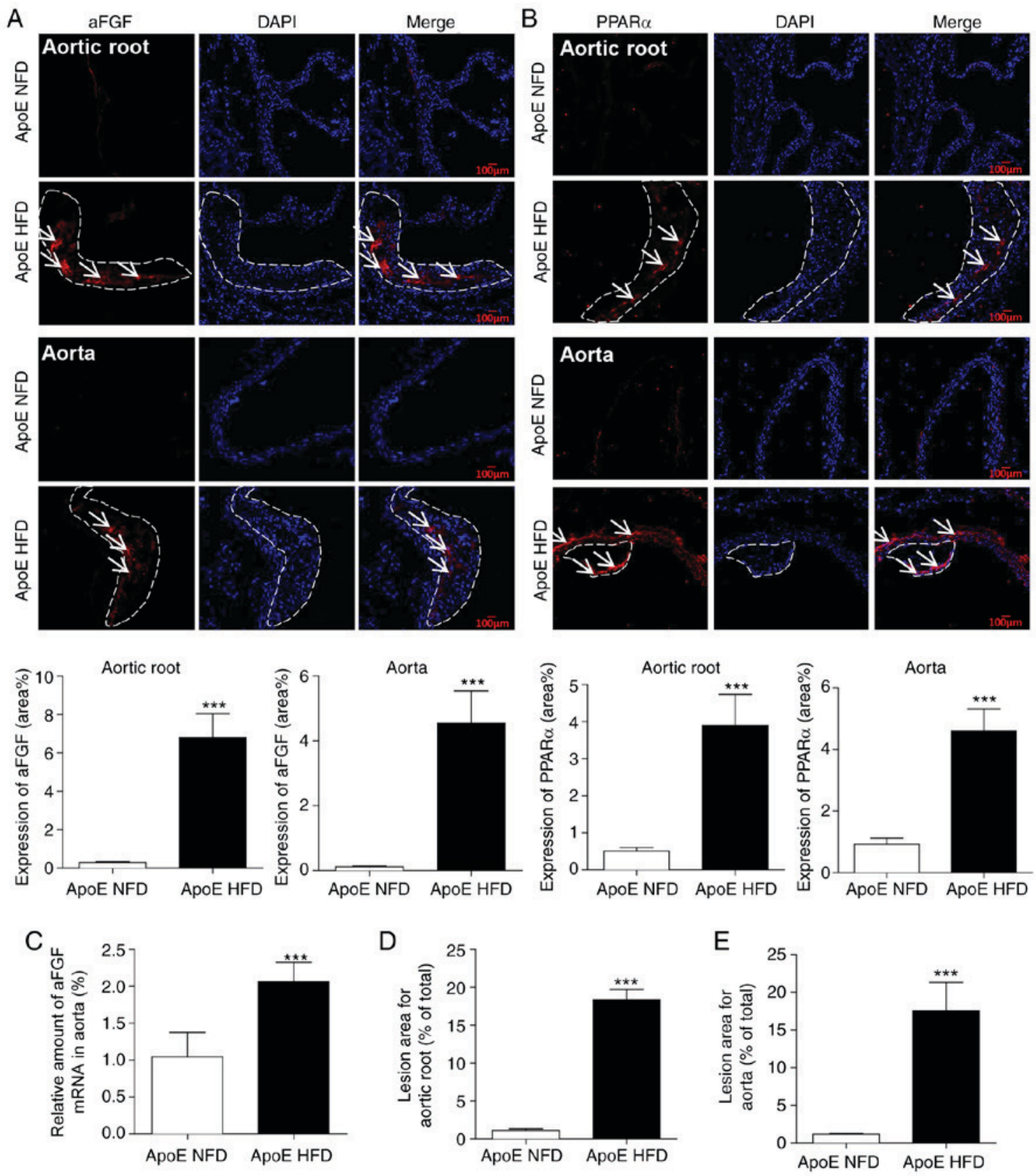

Figure 2. Increased expression of aFGF and PPAR $\alpha$ in the aortic roots and aortas of HFD-fed ApoE ${ }^{-/}$mice. (A) Representative microscopic images of anti-aFGF immunofluorescence staining in the aortic root (upper panels) and the aorta (lower panels). The bar graphs demonstrate the quantification of aFGF expression. (B) Representative microscopic images of anti-PPAR $\alpha$ immunofluorescence staining in the aortic root (upper panels) and the aorta (lower panels). The bar graphs demonstrate the quantification of PPAR $\alpha$ expression. The white arrows indicate the positively stained areas. Scale bar, $100 \mu \mathrm{m}$. (C) Reverse transcription-quantitative polymerase chain reaction analysis of PPAR $\alpha$. Quantification of the atherosclerotic plaque lesion area in (D) the aortic root and (E) the aorta. $n=7 /$ group. ${ }^{* * *} \mathrm{P}<0.001$ vs. respective ApoE NFD. aFGF, acid fibroblast growth factor 1 ; HFD, high-fat diet; PPAR $\alpha$, peroxisome proliferator-activated receptor $\alpha$; ApoE, apolipoprotein E; NFD, normal-fat diet. 

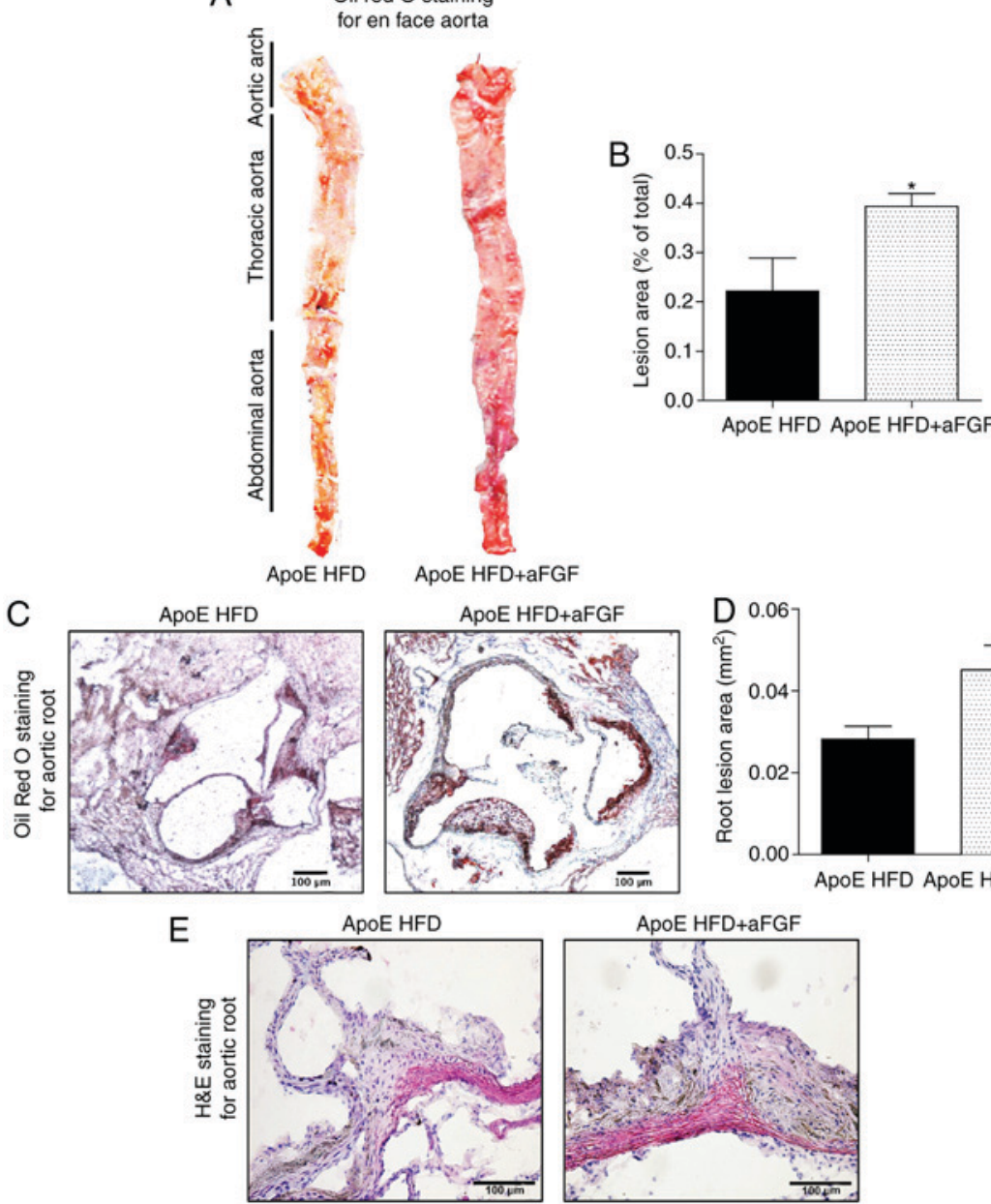

Figure 3. aFGF treatment aggravates atherosclerotic plaque development in HFD-fed $\mathrm{ApoE}^{-/}$mice. ApoE $\mathrm{E}^{-/}$mice were fed a HFD for 8 weeks and treated with aFGF for 8 weeks via intraperitoneal injection. (A) Microphotographs of aortas in the en face preparation following staining with Oil Red O and (B) quantification of the lesion area. (C) Representative examples of cross sections from the Oil Red O-stained aortic root and (D) quantification of the aortic root lesion areas. (E) Representative examples of cross sections from the H\&E stained aortic root. $\mathrm{n}=7 /$ group. Scale bar, $100 \mu \mathrm{m}$. " $\mathrm{P}<0.05$, ${ }^{* *} \mathrm{P}<0.01 \mathrm{vs}$. respective ApoE HFD. aFGF, acid fibroblast growth factor; HFD, high fat diet; H\&E, hematoxylin and eosin; ApoE, apolipoprotein E.

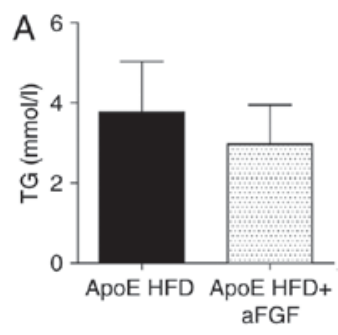

$E$

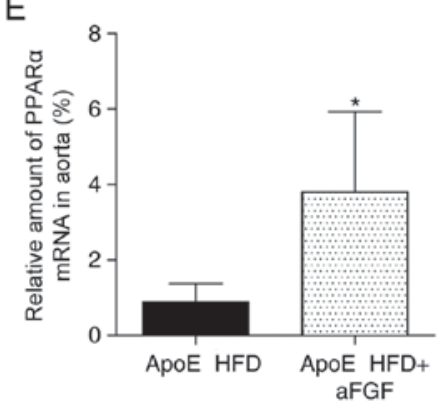

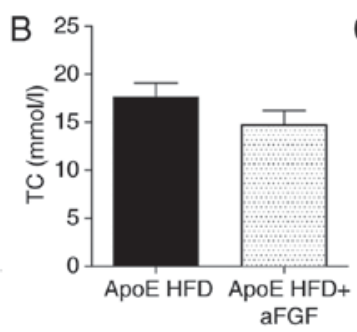

$\mathrm{F}$

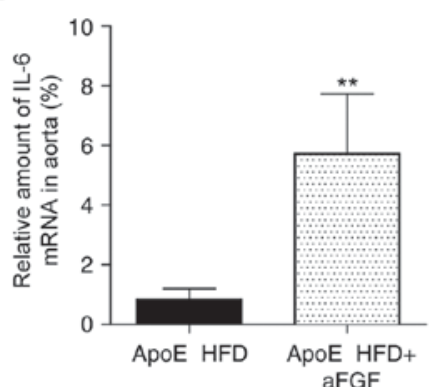

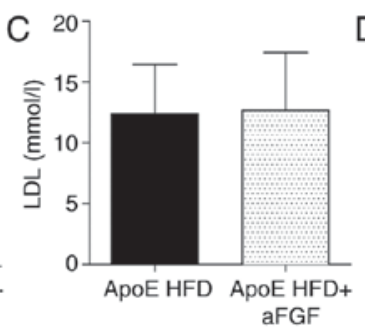



G

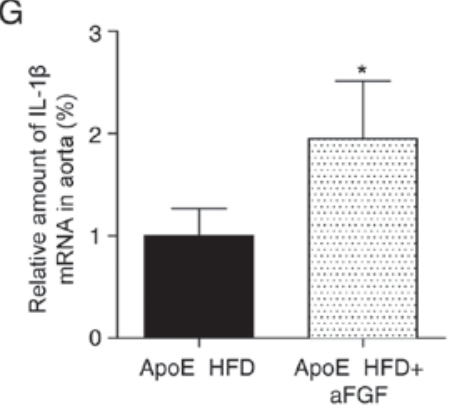

Figure 4. Administration of aFGF increases the mRNA expression levels of PPAR $\alpha$ and inflammatory factors in HFD-fed ApoE $\mathrm{E}^{-/}$mice. Serum expression levels of (A) TG, (B) TC, (C) LDL and (D) HDL. Reverse-transcription-quantitative polymerase chain reaction analysis of (E) PPAR $\alpha$, (F) IL-1 (G) and IL-6. $\mathrm{n}=7 /$ group. ${ }^{*} \mathrm{P}<0.05,{ }^{* *} \mathrm{P}<0.01$ vs. respective ApoE HFD. TG, triglycerides; TC, total cholesterol; LDL, low-density lipoprotein; HDL, high-density lipoprotein; PPAR $\alpha$, peroxisome proliferator-activated receptor $\alpha$; IL, interleukin; ApoE, apolipoprotein E; HFD, high-fat diet. 
that the inhibition of FGFR tyrosine kinase activity reduced atherosclerotic plaque development, suggesting that an active aFGF/FGFR1 signaling system promotes atherosclerosis development.

Tordjman et al (7) demonstrated that PPAR $\alpha$ deficiency reduces insulin resistance and atherosclerosis in ApoE-null mice. The present results additionally demonstrated that the expression levels of PPAR $\alpha$ were increased in aortic atherosclerotic lesions in HFD-fed mice. Although certain evidence suggests a role for aFGF in PPAR- $\gamma$-associated chronic metabolic disease $(1,2)$, the association between aFGF and PPAR $\alpha$ remains unknown. The present results demonstrated that treatment with aFGF increased the mRNA expression levels of PPAR $\alpha$ and inflammatory factors. Therefore, the present results suggested that aFGF may be the upstream regulator of PPAR $\alpha$ and its associated inflammation, which requires validation in future studies.

In conclusion, the present results demonstrated that aFGF promotes the progression of atherosclerotic plaques via PPAR $\alpha$ and inflammatory mechanisms, which occurs independently from alterations in serum lipid expression levels. The present results suggested that targeting aFGF may have therapeutic potential for preventing atherosclerosis.

\section{Acknowledgements}

The authors thank Dr Jibo Han and Dr Xiong Chen. They are the guarantors of the present study and had full access to all the data in the present study, and take responsibility for the integrity of the data and the accuracy of the data analysis.

\section{Funding}

The present study was supported by Public Welfare Science and Technology Program of Wenzhou City (grant no. Y20160306; Wenzhou, China).

\section{Availability of data and materials}

The datasets used and/or analyzed during the current study are available from the corresponding author on reasonable request.

\section{Authors' contributions}

LJ, JX and YD performed the research. LW, XC and JH designed the research study. XC and JH contributed essential reagents or tools. LJ, JX, YD and JH analyzed the data. LW and $\mathrm{JH}$ wrote the manuscript. All authors read and approved the final manuscript.

\section{Ethics approval and consent to participate}

The protocols used for all animal studies in the present study were approved by the Wenzhou Medical University Animal Policy and Welfare Committee (Wenzhou, China; approval no. wydw2014-0058).

\section{Consent for publication}

Not applicable.

\section{Competing interests}

The authors declare that they have no competing interests.

\section{References}

1. Jonker JW, Suh JM, Atkins AR, Ahmadian M, Li P, Whyte J, He M, Juguilon H, Yin YQ, Phillips CT, et al: A PPAR $\gamma$-FGF1 axis is required for adaptive adipose remodelling and metabolic homeostasis. Nature 485: 391-394, 2012.

2. Liu W, Struik D, Nies VJ, Jurdzinski A, Harkema L, de Bruin A, Verkade HJ, Downes M, Evans RM, van Zutphen T and Jonker JW: Effective treatment of steatosis and steatohepatitis by fibroblast growth factor 1 in mouse models of nonalcoholic fatty liver disease. Proc Natl Acad Sci USA 113: 2288-2293, 2016.

3. Pearson-Stuttard J, Guzman-Castillo M, Penalvo JL, Rehm CD, Afshin A, Danaei G, Kypridemos C, Gaziano T, Mozaffarian D, Capewell S and O'Flaherty M: Modeling future cardiovascular disease mortality in the United States: National trends and racial and ethnic disparities. Circulation 133: 967-978, 2016.

4. Daugherty A, Tall AR, Daemen MJAP, Falk E, Fisher EA, García-Cardeña G, Lusis AJ, Owens AP III, Rosenfeld ME, Virmani R, et al: Recommendation on design, execution, and reporting of animal atherosclerosis studies: A scientific statement from the American heart association. Arterioscler Thromb Vasc Biol 37: e131-e157, 2017.

5. Brogi E, Winkles JA, Underwood R, Clinton SK, Alberts GF and Libby P: Distinct patterns of expression of fibroblast growth factors and their receptors in human atheroma and nonatherosclerotic arteries. Association of acidic FGF with plaque microvessels and macrophages. J Clin Invest 92: 2408-2418, 1993.

6. Liau G, Winkles JA, Cannon MS, Kuo L and Chilian WM: Dietary-induced atherosclerotic lesions have increased levels of acidic FGF mRNA and altered cytoskeletal and extracellular matrix mRNA expression. J Vasc Res 30: 327-332, 1993.

7. Tordjman K, Bernal-Mizrachi C, Zemany L, Weng S, Feng C, Zhang F, Leone TC, Coleman T, Kelly DP and Semenkovich CF: PPARalpha deficiency reduces insulin resistance and atherosclerosis in apoE-null mice. J Clin Invest 107: 1025-1034, 2001.

8. Wang L, Huang Z, Huang W, Chen X, Shan P, Zhong P, Khan Z, Wang J, Fang Q, Liang G and Wang Y: Inhibition of epidermal growth factor receptor attenuates atherosclerosis via decreasing inflammation and oxidative stress. Sci Rep 8: 45917, 2017.

9. Lin Y, Bai L, Chen Y, Zhu N, Bai Y, Li Q, Zhao S, Fan J and Liu E: Practical assessment of the quantification of atherosclerotic lesions in apoE-/- mice. Mol Med Rep 12: 5298-5306, 2015.

10. Livak KJ and Schmittgen TD: Analysis of relative gene expression data using real-time quantitative PCR and the 2(-Delta Delta C(T)) method. Methods 25: 402-408, 2001.

11. Sorci-Thomas MG and Thomas MJ: Microdomains, inflammation, and atherosclerosis. Circ Res 118: 679-691, 2016.

12. Pawlak M, Lefebvre P and Staels B: Molecular mechanism of PPAR $\alpha$ action and its impact on lipid metabolism, inflammation and fibrosis in non-alcoholic fatty liver disease. J Hepatol 62: 720-733, 2015.

13. Libby P, Ridker PM and Hansson GK: Progress and challenges in translating the biology of atherosclerosis. Nature 473: 317-325, 2011.

14. Raj T, Kanellakis P, Pomilio G, Jennings G, Bobik A and Agrotis A: Inhibition of fibroblast growth factor receptor signaling attenuates atherosclerosis in apolipoprotein E-deficient mice. Arterioscler Thromb Vasc Biol 26: 1845-1851, 2006.

15. Beenken A and Mohammadi M: The FGF family: Biology, pathophysiology and therapy. Nat Rev Drug Discov 8: 235-253, 2009.

16. Cordon-Cardo C, Vlodavsky I, Haimovitz-Friedman A, Hicklin D and Fuks Z: Expression of basic fibroblast growth factor in normal human tissues. Lab Invest 63: 832-840, 1990.

17. Sigala F, Savvari P, Liontos M, Sigalas P, Pateras IS, Papalampros A, Basdra EK, Kolettas E, Papavassiliou AG and Gorgoulis VG: Increased expression of bFGF is associated with carotid atherosclerotic plaques instability engaging the NF- $\mathrm{B}$ pathway. J Cell Mol Med 14: 2273-2280, 2010.

18. Lin Z, Pan X, Wu F, Ye D, Zhang Y, Wang Y, Jin L, Lian Q, Huang Y, Ding H, et al: Fibroblast growth factor 21 prevents atherosclerosis by suppression of hepatic sterol regulatory element-binding protein-2 and induction of adiponectin in mice. Circulation 131: 1861-1871, 2015. 